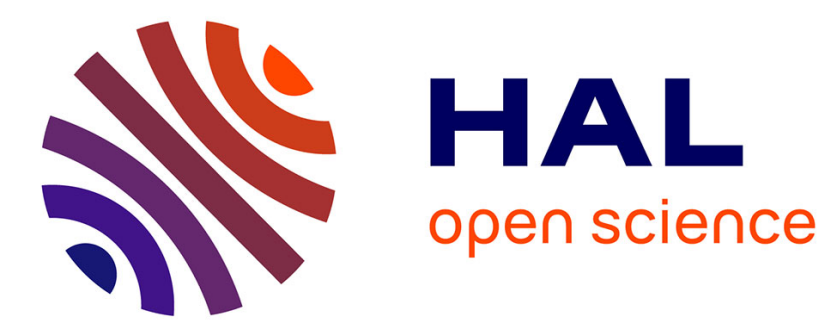

\title{
Spectral risk measures and portfolio selection
}

Alexandre Adam, Mohamed Houkari, Jean-Paul Laurent

\section{To cite this version:}

Alexandre Adam, Mohamed Houkari, Jean-Paul Laurent. Spectral risk measures and portfolio selection. 2007. hal-00165641

\section{HAL Id: hal-00165641 \\ https://hal.science/hal-00165641}

Preprint submitted on 26 Jul 2007

HAL is a multi-disciplinary open access archive for the deposit and dissemination of scientific research documents, whether they are published or not. The documents may come from teaching and research institutions in France or abroad, or from public or private research centers.
L'archive ouverte pluridisciplinaire HAL, est destinée au dépôt et à la diffusion de documents scientifiques de niveau recherche, publiés ou non, émanant des établissements d'enseignement et de recherche français ou étrangers, des laboratoires publics ou privés. 


\section{Institut de Science Financière et d'Assurances}

Les Cahiers de Recherche de I'ISFA

SPECTRAL RISK MEASURES AND PORTFOLIO SELECTION.

Alexandre Adam,

Mohamed Houkari,

Jean-Paul Laurent.

Cahier de recherche WP 2037 (2007)

Université Claude Bernard Lyon 1

Retrouvez la liste complète des cahiers de recherche de l'ISFA à l'adresse :

http://isfaserveur.univ-lyon1.fr/cahiers-recherche-isfa/ 


\title{
Spectral risk measures and portfolio selection ${ }^{1}$
}

This version: July, 19th 2007

\author{
Alexandre ADAM a , Mohamed HOUKARI ${ }^{\text {a, }}$, Jean-Paul LAURENT ${ }^{\text {a, }, \text {, * }}$ \\ ${ }^{a}$ Financial Models, Group ALM, BNP Paribas, 3 rue d'Antin, 75078 Paris Cedex 02, France \\ ${ }^{b}$ ISFA Actuarial School, Université Lyon 1, 50, avenue Tony Garnier, 69007, Lyon, France
}

\begin{abstract}
This paper deals with risk measurement and portfolio optimization under risk constraints. Firstly we give an overview of risk assessment from the viewpoint of risk theory, focusing on moment-based, distortion and spectral risk measures. We subsequently apply these ideas to an asset management framework using a database of hedge funds returns chosen for their nonGaussian features. We deal with the problem of portfolio optimization under risk constraints and lead a comparative analysis of efficient portfolios. We show some robustness of optimal portfolios with respect to the choice of risk measure. Unsurprisingly, risk measures that emphasize large losses lead to slightly more diversified portfolios. However, risk measures that account primarily for worst case scenarios overweight funds with smaller tails which mitigates the relevance of diversification.
\end{abstract}

JEL Classification: C61 ; D81 ; D84 ; G11

Keywords: portfolio selection; expected shortfall; distortion risk measures; spectral risk measures; hedge funds

\section{Introduction and context}

Since the earlier work on expected utility, the axiomatic approach to risk theory has expanded dramatically as illustrated by Yaari (1987), Panjer et al. (1997), Artzner et al. (1999), De Giorgi (2005) among others or the books by Embrechts et al. (2005) or by Denuit et al. (2006). This enables to represent a large set of behaviours towards risk. Following Markowitz (1952), moment-based risk measures were firstly considered. Lately, Acerbi (2002) and Acerbi and Tasche (2002) studied spectral risk measures which involve a weighted average of the quantiles of the loss. The dual theory of choice under risk leads to the class of distortion risk measures (see Yaari (1987) and Wang (2000)). In order to quantify risk, instead of modifying returns as in the expected utility framework, distortion risk measures modify the

\footnotetext{
${ }^{1}$ The content of this paper does not necessarily reflect opinions of BNP Paribas.

${ }^{*}$ Corresponding author.

E-mail addresses: alexandre.adam@,bnpparibas.com, mohamed.houkari@,bnpparibas.com, laurent.jeanpaul@,free.fr.
} 
probability distribution. This approach towards risk can be related to investor's psychology as in Kahneman and Tversky (1979). Other risk measures have been used in a portfolio selection context, such as conditional drawdown (see Cheklov, Uryasev and Zabarankin (2005)).

Rockafellar and Uryasev (2000), Acerbi and Simonetti (2002) and Bassett et al. (2004) have demonstrated the usefulness of efficient linear programming techniques when dealing with spectral or distortion risk measures. This paves the way for a thorough analysis of optimal portfolios and their reliance on the choice of risk measure. Meanwhile, despite some theoretical drawbacks, the computation of mean-variance efficient frontiers remains a cornerstone of quantitative asset management. Whenever returns are Gaussian, one could quite well rely upon the mean-variance framework and the choice of a risk measure is purposeless. Since it is well reported that many asset classes such as hedge funds (see Brooks and Kat (2002)) exhibit non Gaussian returns, especially when considering marginal distributions and individual returns, the robustness of portfolio allocation with respect to the choice of risk measure is thus an important issue.

A general theory involving any arbitrary portfolio distributions and risk measures seems out of scope. We think that it is more insightful to consider a realistic case study, where portfolio returns are actually non Gaussian. One could either rely upon a dynamic statistical model with non Gaussian effects as in Giamouridis and Vrontos (2007) or Morton et al. (2006). Another possibility is to use a set of historical returns and the empirical measure as in Heyde et al. (2006). We will thereafter follow this route, though there are obviously some various issues to be considered with respect to the estimation of risk measures (see Scaillet (2004), Gouriéroux and Liu (2006)). On practical grounds, Krokhmal et al. (2002), Chabaane et al. (2006) studied the dependence of optimal portfolios with the choice of risk measures. This paper extends previous results to spectral and distortion risk measures.

The paper is organized as follows. In Section 2 we present and discuss some useful risk measures - moment based and spectral/distortion risk measures - from an asset management point of view. We show how spectral and distortion risk measures are related to VaR and expected shortfall. We also provide a simple characterization of distortion risk measures and recall how this theory is related to the standard expected utility approach. Section 3 deals with the problem of portfolio optimization under a risk measure constraint. The empirical study is based upon a database of hedge funds for which we emphasize the non Gaussian features. We subsequently discuss the differences between the optimal portfolios. Risk measures that emphasize large losses lead to slightly more diversified portfolios. However, risk measures that account primarily for worst case scenarios overweight funds with smaller tails which mitigates the relevance of diversification.

\section{Theoretical overview of risk measures for asset management}

The purpose of this section is to discuss some risk measures from the viewpoint of an asset manager. We will consider portfolio returns over a given time horizon: other risk management approaches rather deal with wealth or with the increment of wealth, but portfolio returns are easier to grasp in an asset management framework. As for the time horizon, we will thereafter deal with a one month time horizon, corresponding to the periodicity of our dataset. Dealing with longer horizons would not involve only the modelling of conditional means and variances of returns, as usually required in a mean-variance framework, but of the entire distribution. Furthermore, even if returns were independent, the convolution of discrete distributions would lead to large dimensional problems and this is likely to dramatically increase numerical difficulties. 


\subsection{Axiomatic approach to risk measurement}

From a mathematical point of view, we will be given a finite ${ }^{2}$ probability space $(\Omega, \mathrm{A}, P)$. Given a portfolio return $X$, we denote by $F_{X}$, the corresponding distribution function: $x \in \mathbb{R} \rightarrow F_{X}(x)=P(X \leq x)$. A risk measure is a functional over the set of random portfolio returns $X \rightarrow \rho(X) \in \mathbb{R}$. It may fulfil some axioms such as:

- H1 Positive Homogeneity: for every random portfolio return $X$ and real value $\lambda>0$, $\rho(\lambda X)=\lambda \rho(X)$;

- H2 Translation-Invariance: for every random portfolio return $X$ and real value $\alpha$, $\rho(X+\alpha)=\rho(X)-\alpha$;

- H3 Monotonicity: for every random portfolio returns $X$ and $Y$ such that $X \geq Y$, $\rho(X) \leq \rho(Y)$;

- H4 Sub-additivity: for every random portfolio returns $X$ and $Y$, $\rho(X+Y) \leq \rho(X)+\rho(Y)$;

- H5 Law-Invariance: for every random portfolio returns $X$ and $Y$ with distribution functions $F_{X}$ and $F_{Y}, F_{X}=F_{Y} \Rightarrow \rho(X)=\rho(Y)$;

- H6 Comonotonic ${ }^{3}$ Additivity: for every comonotonic random variables $X$ and $Y$, $\rho(X+Y)=\rho(X)+\rho(Y)$.

Coherent measures of risk as discussed in Artzner et al. (1999) fulfil H1 to H4, distortion risk measures as defined in Panjer et al. (1997) comply with H1, H2, H3, H5, H6, while spectral risk measures considered by Acerbi (2002) conform to all the above axioms. We also refer to Rockafellar et al. (2006) for discussion about deviation risk measures. Let us remark that from $\mathrm{H} 1, \rho(0)=0$; thus, from $\mathrm{H} 2, \rho(r)=-r$, where $r$ is a risk-free return. From $\mathrm{H} 2$ and $\mathrm{H} 3$, $\rho(X) \leq-\inf X$. All the risk measures being considered thereafter are positive homogeneous.

\subsection{Use of the empirical measure}

We will thereafter consider a set of historical funds returns $\left\{r_{1}, \ldots, r_{n}\right\}$ as a realization of an $m$-dimensional strictly stationary process $R=\left(R_{t}\right)_{1 \leq t \leq n} . R_{t}^{k}$ denotes the return on fund $k$ at date $t$ and each $R_{t}=\left(R_{t}^{1}, \ldots, R_{t}^{m}\right)$ represents the $m$ returns at date $t$. We will consider portfolios based on this pool of funds, defined by their allocation vector $a=\left(a_{1}, \ldots, a_{m}\right) \in \mathbb{R}^{m}$ and the related return process $\Pi(a)=\left(\Pi(a)_{t}=\sum_{k=1}^{m} a_{k} R_{t}^{k}\right)_{1 \leq t \leq n}$. Then the realization of this return process is given by $\left\{\pi(a)_{t}=\sum_{k=1}^{m} a_{k} r_{t}^{k}, 1 \leq t \leq n\right\}$.

\footnotetext{
${ }^{2}$ This simplifies the mathematical framework. See Delbaen (2002) for issues related to more general probability spaces.

${ }^{3}$ Comonotonicity is related to perfect dependence. Two random variables $X$ and $Y$ are comonotonic if: $\forall\left(\omega_{1}, \omega_{2}\right) \in \Omega^{2},\left(X\left(\omega_{2}\right)-X\left(\omega_{1}\right)\right)\left(Y\left(\omega_{2}\right)-Y\left(\omega_{1}\right)\right) \geq 0$.
} 
Given a set of asset returns associated with a random vector $R$ taking values in $\mathbb{R}^{m}$, we can think of using the law of $R, P_{R}$ and deal with the space $\left(\mathbb{R}^{m}, B\left(\mathbb{R}^{m}\right), P_{R}\right)$. We believe that it is more intuitive to work with the latter space and therefore we further restrict to law-invariant risk measures, which is actually the case in all our examples.

In an asset management framework, we can only rely upon historical databases and do not have a direct access to the true probability $P_{R}$. There is thus clearly an inference issue and we can only deal with an estimator $\hat{P}_{R}$. At this stage, we can think of parametric or nonparametric approaches.

Regarding parametric approaches, while there are obviously many dynamic models of individual asset returns to be considered, there are also various ways to address the multivariate dependence structure. As discussed above, relying upon a simple conditional Gaussian distribution or any other arbitrary dependence structure involving some elliptical distribution is irrelevant. In our view, since we want to emphasize non Gaussian effects through a reasonable case study, we will leave aside the intricate issue of multivariate time dependence and eventually work with marginal distributions ${ }^{4}$.

While the use of nonparametric approaches is more flexible ${ }^{5}$, we could either rely upon the empirical measure (more generally upon $L$ - estimators as in Granger and Silvapulle (2001)) or upon kernel based estimators (see Gouriéroux et al. (2000), Scaillet (2004), Gouriéroux and Liu (2006)). The main differences regarding the computation of risk measures and optimal portfolios are the following: using the empirical measure involves a finite state space; empirical risk measures are not differentiable with respect to portfolio allocation (see Laurent (2003)) but one can rely upon very efficient linear programming techniques to derive optimal portfolios as in Rockafellar and Uryasev (2000). When using kernel based estimators, risk measures usually become differentiable with respect to portfolio allocation; efficient computations of risk sensitivities can be achieved leading to steepest descent optimisation techniques. As for theoretical properties of the estimators involved in the two approaches, we refer to Scaillet (2004) and Gouriéroux and Liu (2006). Chabaane et al. (2006) studied the dependence of portfolio choice with respect to the choice of Value at Risk estimator and found only small effects ${ }^{6}$.

In this paper, we will stay in line with the classical work of Rockafellar and Uryasev (2000), dealing with portfolio selection under expected shortfall constraints, and thus $\hat{P}_{R}$ will be the empirical measure. Since $\hat{P}_{R}$ fulfils the permutation invariance axiom, the risk measures further considered belong to the class of "natural risk statistics" (see Heyde et al. (2006)). As seen below in our case study, we think that some of the features exhibited might reasonably be extended to $L$ - estimators.

\footnotetext{
${ }^{4}$ It is well-known that marginal distributions tend to exhibit fatter tails than conditional distributions.

${ }^{5}$ We refer to Morton et al. (2006) for a semiparametric approach involving a Gaussian copula.

${ }^{6}$ Since spectral risk measures can be viewed as weighted averages of Values at Risk, we can think that the choice of the nonparametric procedure is not of first importance; see Scaillet (2004) for a discussion in the case of the Expected Shortfall.
} 
From now on, a random variable $X$ will be related to some portfolio allocation $a$ and the corresponding historical returns are denoted by $\left\{x_{1}, \ldots, x_{n}\right\}$. The empirical distribution function is given by: $x \in \mathbb{R} \rightarrow \hat{F}_{X}(x)=\frac{1}{n} \sum_{i=1}^{n} 1_{\left[x_{i}, \infty[\right.}(x)$. Since only empirical estimators of the distribution functions will be further involved, for simplicity $F_{X}$ will state for $\hat{F}_{X}$. Besides, as we deal with empirical estimators, all being related to the historical realisations $\left\{r_{1}, \ldots, r_{n}\right\}$ of the asset returns, the probabilistic framework behaves as if the state space $\Omega$ were finite with $\operatorname{card}(\Omega)=n$. Thus this will be our assumption from now on, setting our probabilistic framework as in Artzner et al. (1999).

We will further consider the (lower) quantile function:

$$
\alpha \in] 0,1] \rightarrow F_{X}^{-1}(\alpha)=\inf \left\{x \mid F_{X}(x) \geq \alpha\right\},
$$

where $\inf \varnothing=+\infty$. We denote by $\left\{x_{1: n}, \ldots, x_{n: n}\right\}$ the set of ordered historical portfolio returns, $x_{1: n} \leq \ldots \leq x_{n: n}$. The (empirical) quantile function is such that $F_{X}^{-1}(\alpha)=x_{i: n}$ for $\alpha \in\left(\frac{i-1}{n}, \frac{i}{n}\right]$, $i=1, \ldots, n$. We can also write $F_{X}^{-1}(\alpha)=x_{\lceil n \alpha\rceil: n}, 0<\alpha<1$, where $\lceil n \alpha\rceil$ is the smallest integer above $n \alpha$.

\subsection{Moment based risk measures}

Markowitz (1952) standard deviation is the most famous one, as it gave birth to asset management strategies in the fifties and still constitutes a benchmark in these activities. This risk measure can be extended to higher moments as illustrated in a portfolio selection context by Davies et al. (2003). However, in addition to its symmetric behaviour towards profits and losses, standard deviation fails to be translation invariant, monotonic and comonotonic additive and can exceed the maximal loss. For this reason, we may consider the class of onesided moment based risk measures as discussed in Fischer (2003):

$$
\rho_{q, a}(X)=-E^{P}[X]+a\left(E^{P}\left[\left(X-E^{P}[X]\right)_{-}^{q}\right]\right)^{1 / q}=-\bar{x}+a\left(\frac{1}{n} \sum_{i=1}^{n}\left(x_{i}-\bar{x}\right)_{-}^{q}\right)^{1 / q},
$$

where $E^{P}[X]=\frac{1}{n} \sum_{i=1}^{n} x_{i}=\bar{x}, Z_{-}=\max (-Z, 0), 0 \leq a \leq 1$ and $1 \leq q \leq \infty$. It can be shown that these risk measures are coherent and law-invariant; however, they fail to be comonotonic additive. We will further investigate the cases $q=1,2$. $q=1$ corresponds to the one-sided absolute deviation from the mean and has been used in Denneberg (1990) and Krokhmal et al. (2002). $q=2$ is associated with the well-known lower semi-variance. Denneberg (1990) also considered risk measures based upon the absolute deviation from the median $F_{X}^{-1}(1 / 2)$ :

$$
\rho_{a}(X)=-E^{P}[X]+a E^{P}\left[\left|X-F_{X}^{-1}(1 / 2)\right|\right]=-\frac{1}{n} \sum_{i=1}^{n}\left(x_{i: n}+a\left|x_{i: n}-x_{\lceil n / 2\rceil: n}\right|\right),
$$

where $0 \leq a \leq 1$. As for the risk measures based on one sided moments, these measures are coherent and law-invariant. Using the median instead of the expectation makes them comonotonic additive. Moreover, Denneberg shows that the risk measure can be simply deduced from the quantile function as: 


$$
\rho_{a}(X)=-\int_{0}^{1} \phi(p) F_{X}^{-1}(p) d p
$$

where $\phi(p)=(1+a) 1_{[0,1 / 2)}(p)+(1-a) 1_{[1 / 2,1]}(p)$.

Let us remark that the previous risk measures are more pessimistic than the expectation, i.e. they fulfil $\rho(X) \geq-E^{P}[X]$.

\subsection{VaR and expected shortfall}

A typical example of a popular risk measure is the Value-at-Risk (or VaR). The Value-at-Risk at a specified threshold $\alpha$ is defined as:

$$
\operatorname{VaR}_{\alpha}(X)=-F_{X}^{-1}(\alpha)=-\inf \left\{x \mid F_{X}(x) \geq \alpha\right\}=-x_{\lceil n \alpha\rceil: n} .
$$

Thus, Values at Risk are associated to ordered statistics of portfolio returns. VaR fulfils H1, H2, H3, H5 and H6 (see for example Dhaene et al. (2002) for the fulfilment of comonotonic additivity) but fails to comply with the sub-additivity property and thus does not provide good incentives with respect to portfolio diversification.

The expected shortfall (see Acerbi and Tasche (2002), or Rockafellar and Uryasev (2002)) is a typical example of a coherent risk measure based upon quantiles of the returns. The expected shortfall at level $\alpha$ can be expressed as follows:

$$
\operatorname{ES}_{\alpha}(X)=-\frac{1}{\alpha} \int_{0}^{\alpha} F_{X}^{-1}(p) d p
$$

Considering, as before, the empirical distribution function of $X$ and since $F_{X}^{-1}(p)=x_{\lceil n p\rceil n}$, we also have:

$$
\operatorname{ES}_{\alpha}(X)=-\frac{1}{n \alpha}\left(\sum_{i=1}^{\lceil n \alpha\rceil-1} x_{i: n}+(n \alpha-\lceil n \alpha\rceil+1) x_{\lceil n \alpha\rceil: n}\right) .
$$

Thus, the expected shortfall is a weighted average of ordered statistics and can be easily computed from historical portfolio returns ${ }^{7}$.

From the above expression, it can be easily checked that $\alpha \in(0,1] \rightarrow \operatorname{ES}_{\alpha}(X)$ is continuous and non-increasing. The extreme cases correspond to $\operatorname{ES}_{1}(X)=-E^{P}[X]$ which is the less pessimistic risk measure and $\lim _{\alpha \rightarrow 0} \operatorname{ES}_{\alpha}(X)=-x_{1: n}$ associated with the worst case scenario.

Unlike Value-at-Risk, the expected shortfall is a coherent risk measure. In fact, it is the smallest coherent, comonotonic additive and law-invariant risk measure which dominates

\footnotetext{
${ }^{7}$ Since we deal with historical returns and the empirical distribution function, we may wonder whether the expected shortfall computed under the empirical measure converges, as the number of observations tends to infinity, to the expected shortfall under the true distribution function. This is fortunately the case; we refer to Acerbi and Tasche (2002) or Jones and Zitikis (2003) for a more thorough discussion.
} 
$\mathrm{VaR}$ as shown in Dhaene et al. (2004). Therefore the expected shortfall can be seen as a "natural" coherent extension of the Value-at-Risk ${ }^{8}$.

Following the work of Artzner et al. (1999) any coherent risk measure defined on a finite state space $\Omega$ can be set under the following form:

$$
\rho(X)=-\inf \left\{E^{Q}[X] \mid Q \in \Pi\right\}
$$

where $\Pi$ is a set of probability measures $Q$ defined on $\Omega$. In the case of the expected shortfall at level $\alpha$, we can indeed fully characterize the set $\Pi_{\alpha}$ such that:

$$
\operatorname{ES}_{\alpha}(X)=-\inf \left\{E^{Q}[X] \mid Q \in \Pi_{\alpha}\right\},
$$

(see Proposition A.1 in Appendix A). $\Pi_{\alpha}$ is obtained by considering scenarios that put a constant weight of $\frac{1}{n \alpha}$ upon $\lceil n \alpha\rceil$ events and 0 upon the others.

The use of expected shortfall is consistent with standard microeconomic theory thanks to the following result, whose proof is given in Appendix B:

$$
X \underset{S O S D}{\leq} Y \Leftrightarrow \forall \alpha \in(0,1], \operatorname{ES}_{\alpha}(X) \geq \operatorname{ES}_{\alpha}(Y) .
$$

We recall that $X$ is smaller than $Y$ with respect to second order stochastic dominance and we denote $X \underset{S O S D}{\leq} Y$, if for every non-decreasing concave function $u$ such that the expectations are defined we have $E^{P}[u(X)] \leq E^{P}[u(Y)]$. Thus, any Von Neumann-Morgenstern investor would prefer $Y$ to $X$.

\subsection{Spectral risk measures}

In this subsection, we deal with spectral risk measures, a class of measures based on integrals of the quantile function of the portfolio return. A spectral risk measure consists in a weighted average of the quantiles of the distribution of the returns using a non-increasing weight function called the spectrum. It is defined as follows:

$$
M_{\phi}(X)=-\int_{0}^{1} \phi(p) F_{X}^{-1}(p) d p,
$$

where $\phi$ is a non-negative, non-increasing, right-continuous integrable function defined on $[0,1]$ and such that $\int_{0}^{1} \phi(p) d p=1$. Kusuoka $(2001)^{9}$ showed that spectral risk measures coincide with coherent, comonotonic-additive and law-invariant risk measures. In other

${ }^{8}$ The expected shortfall can be further connected to the VaR since for a continuous distribution of portfolio returns, it coincides with the (lower) Tail Conditional Expectation as stated by Corollary 5.3 in Acerbi and Tasche (2002):

$$
\operatorname{ES}_{\alpha}(X)=-E^{P}\left[X \mid X \leq-\operatorname{VaR}_{\alpha}(X)\right] \text {. }
$$

The latter expression, defining the lower Tail Conditional Expectation, corresponds to the average value of the returns on a short position beyond VaR at level $\alpha$.

${ }^{9}$ See also Tasche (2002) or Jouini et al. (2006), for a thorough discussion about the assumptions. 
words, any risk measure fulfilling assumptions $\mathrm{H} 1$ to $\mathrm{H} 6$ is a spectral risk measure. The coherence of spectral risk measures comes from the assumptions made on the spectrum $\phi$. If any of these assumptions is relaxed, the measure is no longer coherent: note for example that if $\phi$ is no more non-increasing, as it is in the case of VaR, the measure is no longer subadditive. The usefulness of each of the assumptions on $\phi$ is more precisely explained in Acerbi (2002).

Since $F_{X}^{-1}(p)=x_{\lceil n p\rceil: n}$, we can express the spectral risk measure of the empirical distribution of portfolio returns as:

$$
M_{\phi}(X)=-\sum_{i=1}^{n} \lambda_{i} x_{i: n}
$$

where $\lambda_{i}=\int_{(i-1) / n}^{i / n} \phi(p) d p \geq 0, \sum_{i=1}^{n} \lambda_{i}=1$. Then the computation of any spectral risk measure involves an average value of ranked portfolio returns ${ }^{10}$.

The expected shortfall is a spectral risk measure with $\phi(p)=\frac{1}{\alpha} \times 1_{[0, \alpha]}(p)$ and conversely, any spectral risk measure can be expressed as a weighted average of expected shortfalls (see Appendix $\mathrm{C}$ for a proof of this result). As an example, let us turn back to the Denneberg risk measure. It can be seen that $p \rightarrow \phi(p)=(1+a) 1_{[0,1 / 2)}(p)+(1-a) 1_{[1 / 2,1]}(p)$ fulfils the required properties; this means that we are dealing with a spectral risk measure. Indeed, it can be checked that the Denneberg risk measure is equal to $(1-a) \mathrm{ES}_{1}(X)+a \mathrm{ES}_{1 / 2}(X)$.

As in the case of the expected shortfall, we can fully characterize the set $\Pi_{\phi}$ such that:

$$
M_{\phi}(X)=-\inf \left\{E^{Q}[X] \mid Q \in \Pi_{\phi}\right\},
$$

(see Proposition A.2 in Appendix A).

Spectral risk measures are also consistent with expected utility theory and second order stochastic dominance thanks to the following result, whose proof is given in Appendix B:

$$
X \underset{S O S D}{\leq} Y \Leftrightarrow \text { For any spectral risk measure } M_{\phi} \text { we have } M_{\phi}(X) \geq M_{\phi}(Y) \text {. }
$$

When portfolio returns are Gaussian, spectral risk measures are linear with respect to portfolio expected return and standard deviation (see Proposition D.1. in Appendix D). Therefore, in the Gaussian case, as far as portfolio selection is concerned, one could rely upon a meanvariance framework.

\subsection{Distortion risk measures}

${ }^{10}$ As for the expected shortfall, the spectral risk measure computed under the empirical distribution converges to the spectral risk measure computed under the true limit distribution as the number of observations tends to infinity. We refer to Acerbi (2002) for a more thorough discussion. 
Distortion risk measures take their origin in Yaari's Dual theory of choice under risk (1987). Yaari's idea consists in measuring risk by applying a distortion function $f$ on $F_{X}$, the distribution function of the portfolio returns. This formally leads to ${ }^{11}$ :

$$
\rho_{f}(X)=-\int_{-\infty}^{+\infty} x d\left(f \circ F_{X}\right)(x) \stackrel{\Delta}{=}-\int_{-\infty}^{0}\left[1-f\left(1-F_{X}(x)\right)\right] d x+\int_{0}^{+\infty} f\left(1-F_{X}(x)\right) d x,
$$

where the distortion function $f$ is non-decreasing, concave and such that $f(0)=0$ and $f(1)=1$. In other words, the distortion function increases the weight put on negative events and deflates that of positive ones. The case $f=i d$ corresponds to the expected return, $\rho_{i d}(X)=-E^{P}[X]$. Besides, the expected return is the less pessimistic distortion risk measure, since the distortion function, as defined above, verifies $\forall u \in[0,1], f(u) \geq u$, and the definition of distortion risk measures above gives $\rho_{f}(X) \geq \rho_{i d}(X)=-E^{P}[X]$.

The previous definition creates a whole class of risk measures, also known as the set of concave distortion risk measures. Besides, the theory of integration developed in Denneberg (1994) leads to:

$$
\rho_{f}(X)=-\int_{-\infty}^{+\infty} x d\left(f \circ F_{X}\right)(x)=-\int_{0}^{1} \phi(p) F_{X}^{-1}(p) d p=M_{\phi}(X)
$$

with $f(p)=\int_{0}^{p} \phi(u) d u$ for $p \in[0,1]$. This shows the identity between the set of concave distortion and spectral risk measures. As a consequence, any concave distortion risk measure fulfils assumptions $\mathrm{H} 1$ to H6. For example, the expected shortfall at level $\alpha$ corresponds to the spectrum $\phi(p)=\frac{1}{\alpha} \times 1_{[0, \alpha]}(p)$ and to the distortion function $f(p)=\min \left(\frac{p}{\alpha}, 1\right)$, for $p \in[0,1] .{ }^{12}$

Distortion risk measures were firstly applied in an insurance context. For example, Wang (2000) considers the following specification of $f$ :

$$
f(p)=\Phi\left[\Phi^{-1}(p)-\Phi^{-1}(q)\right],
$$

for $p \in[0,1]$, where $0<q \leq 0.5$ is some parameter ${ }^{13}$. The distortion function $f$ is indeed non-decreasing, concave and such that $f(0)=0$ and $f(1)=1$. The corresponding risk measure $W T_{q}$ is known as the Wang-transform.

\footnotetext{
${ }^{11}$ This definition makes reference to the Choquet integral theory as in Denneberg (1994) for example. The concept of $\int g(u) d\left(f \circ F_{X}\right)(u)$ makes sense thanks to the Stieltjes integral which states that the function $f \circ F_{X}$ only has to be non-decreasing and finite.

12 Theorem 9 in Dhaene et al. (2004) characterizes the expected shortfall of $X$-as we define it - as the lower bound of the set of values taken on $X$ by the concave distortion risk measures dominating $\mathrm{VaR}$.

${ }^{13}$ When $q=0.5, f=i d$ and we are back in the case of the less pessimistic risk measure based upon the expectation. We need $q \leq 0.5$ in order to get a concave distortion risk measure.
} 
The parameter $q$ can be changed to make the Wang-transform either sharper on high losses or softer and more receptive to positive returns. For a Gaussian portfolio return $X$, $W T_{q}(X)=-E^{P}[X]-\Phi^{-1}(q) \sigma(X)$. For such a Gaussian portfolio return, with the Value-atRisk, we also have $\operatorname{VaR}_{q}(X)=-E^{P}[X]-\Phi^{-1}(q) \sigma(X)$, which shows the equality between the Wang-transform (at level $q$ ) and the Value-at-Risk (at level $q$ ) for Gaussian returns. Such a property can be used for the calibration of $q$ and may be appealing when integrating the measurement of market risks - where Value-at-Risk is still widely used - and technical risks arising from the insurance liabilities. As a consequence, the Wang transform might be considered also by asset managers within insurance companies.

\section{Portfolio optimization under risk constraints}

\subsection{Framework of the study}

Our study has been performed on a pool of 16 hedge funds. We had access to monthly returns, from January 1990 to July 2001. We used the same database as Chabaane et al. (2006). We give in Table 3.1 some descriptive statistics, including mean (m), standard deviation ( $\mathbf{\sigma})$, skewness (s) and kurtosis (к).

We also computed the hedge funds monthly betas and Jensen's alphas with respect to the S\&P500 index. This basic approach is motivated by Agarwal and Naik (2000) who derive a multi-factor model for hedge funds returns based on 7 factors including the S\&P500 composite index and found some significant market exposure even for non-directional hedge funds. However our low betas and high alphas have to be taken carefully due to survivorship bias. Indeed, hedge funds' performances are known to be over-estimated since their evaluations only deal with survivor funds, as studied in Bares et al. (2001) or Amin and Kat (2003). This has significant influence on the values of the betas and Jensen's alphas. Cvitanic et al. (2003) address the uncertainty of hedge funds alphas as far as portfolio selection is concerned. More accurate assessment of survivorship bias in the hedge funds industry can be found in Brown et al. (1997) or Fung and Hsieh (1997) for example. As for our study, our aim is to use a class of assets which does not follow standard distributions; our pool of hedge funds achieves that, as we further point out.

\begin{tabular}{|c|c|c|c|c|c|c|c|c|}
\hline HF & Hedge Funds & $\mathbf{m}$ & $\boldsymbol{\sigma}$ & $\mathbf{s}$ & $\kappa$ & Sharpe Ratio & Betas & Alphas \\
\hline 1 & AXA Rosenberg & $0,46 \%$ & $2,31 \%$ & 0,17 & 5,92 & $0,20 \%$ & $-14 \%$ & $0,57 \%$ \\
\hline 2 & Discovery MasterFund & $0,51 \%$ & $4,30 \%$ & $-0,06$ & 0,15 & $1,27 \%$ & $2 \%$ & $0,49 \%$ \\
\hline 3 & Aetos Corporation & $0,99 \%$ & $2,34 \%$ & $-0,36$ & 3,40 & $22,88 \%$ & $25 \%$ & $0,79 \%$ \\
\hline 4 & Bennett Restructuring Fund & $1,25 \%$ & $2,15 \%$ & $-0,16$ & 3,22 & $36,81 \%$ & $16 \%$ & $1,11 \%$ \\
\hline 5 & Calamos Convertible Hedge Fund & $0,85 \%$ & $2,33 \%$ & 0,15 & 1,16 & $17,18 \%$ & $37 \%$ & $0,56 \%$ \\
\hline 6 & Sage Capital Limited Partnership & $0,78 \%$ & $0,70 \%$ & $-0,67$ & 1,34 & $47,04 \%$ & $7 \%$ & $0,72 \%$ \\
\hline 7 & Genesis Emerging Markets Fund & $0,84 \%$ & $5,78 \%$ & $-0,70$ & 2,80 & $6,71 \%$ & $78 \%$ & $0,22 \%$ \\
\hline 8 & RXR Secured Participating Note & $0,97 \%$ & $1,86 \%$ & 0,49 & 2,13 & $27,94 \%$ & $21 \%$ & $0,81 \%$ \\
\hline 9 & Arrowsmith Fund & $2,01 \%$ & $7,81 \%$ & 3,05 & 26,20 & $19,89 \%$ & $37 \%$ & $1,71 \%$ \\
\hline 10 & Blue Rock Capital Fund & $0,69 \%$ & $1,00 \%$ & 0,35 & 3,28 & $24,23 \%$ & $9 \%$ & $0,62 \%$ \\
\hline 11 & Dean Witter Cornerstone Fund IV & $1,09 \%$ & $6,69 \%$ & 1,56 & 4,00 & $9,61 \%$ & $-3 \%$ & $1,12 \%$ \\
\hline
\end{tabular}




\begin{tabular}{llrrrrrrr}
12 & GAMut Investments Inc & $1,86 \%$ & $4,16 \%$ & 0,71 & 2,03 & $33,79 \%$ & $6 \%$ & $1,81 \%$ \\
13 & Aquila International Fund & $0,79 \%$ & $4,87 \%$ & $-0,26$ & 1,05 & $6,88 \%$ & $69 \%$ & $0,23 \%$ \\
14 & Bay Capital Management & $0,81 \%$ & $5,57 \%$ & 0,41 & 0,35 & $6,37 \%$ & $24 \%$ & $0,61 \%$ \\
15 & Blenheim Investments & $1,28 \%$ & $8,54 \%$ & 0,64 & 4,46 & $9,72 \%$ & $10 \%$ & $1,20 \%$ \\
16 & Red Oak Commodity Advisors & $1,52 \%$ & $8,39 \%$ & 0,41 & 1,56 & $12,69 \%$ & $70 \%$ & $0,96 \%$ \\
\hline
\end{tabular}

Table 3.1. Descriptive analysis of the hedge funds (monthly returns)

In our database, two hedge funds are highly rewarding assets - HF 9 and 12 - therefore we may expect that these two assets - at least HF 9 which is the most rewarding one - should be selected in optimal portfolios when the risk constraint is pretty weak. However HF 9 is associated with a very high kurtosis therefore it should be all the less probably selected as the risk constraint is demanding. On the contrary, HF 6 combines low volatility and high Sharpe ratio and therefore it could be selected when the risk constraint is tight.

On the other hand, as we will further notice, some assets - for example, HF 2 and 11 sometimes appear in efficient portfolios for low risk levels although they are weakly rewarding and highly volatile. The presence of these assets in efficient portfolios is probably due to diversification effects that counterbalance the risk contributions of other assets in the portfolio. We show below the correlation matrix between hedge funds, showing, for example, negative correlation effects between the assets of the pool. However, let us recall that we can combine positive tail dependence and negative linear correlation implying two opposite effects from the viewpoint of diversification.

\begin{tabular}{rrrrrrrrrrrrrrrr}
\hline $\mathbf{1 0 0}$ & 14 & -3 & 0 & -20 & -13 & -33 & -4 & -15 & 5 & 7 & 8 & -24 & -7 & -2 & -28 \\
14 & $\mathbf{1 0 0}$ & -1 & -13 & -5 & -2 & -5 & 6 & -10 & 28 & 11 & -2 & 3 & 2 & -5 & 7 \\
-3 & -1 & $\mathbf{1 0 0}$ & 35 & 35 & 21 & 31 & 12 & 16 & 11 & 1 & -13 & 30 & -5 & 0 & 12 \\
0 & -13 & 35 & $\mathbf{1 0 0}$ & 25 & 31 & 40 & -3 & 16 & -4 & 3 & -8 & 31 & 9 & -4 & 11 \\
-20 & -5 & 35 & 25 & $\mathbf{1 0 0}$ & 40 & 50 & 21 & 18 & 30 & -15 & 5 & 49 & 12 & 1 & 16 \\
-13 & -2 & 21 & 31 & 40 & $\mathbf{1 0 0}$ & 40 & 8 & 14 & 7 & 2 & 1 & 34 & -5 & -2 & 2 \\
-33 & -5 & 31 & 40 & 50 & 40 & $\mathbf{1 0 0}$ & 15 & 22 & 10 & -8 & -9 & 64 & 14 & 16 & 31 \\
-4 & 6 & 12 & -3 & 21 & 8 & 15 & $\mathbf{1 0 0}$ & 8 & 25 & 37 & 41 & 25 & 10 & 11 & 31 \\
-15 & -10 & 16 & 16 & 18 & 14 & 22 & 8 & $\mathbf{1 0 0}$ & 1 & -9 & -1 & 17 & 7 & -1 & 11 \\
5 & 28 & 11 & -4 & 30 & 7 & 10 & 25 & 1 & $\mathbf{1 0 0}$ & 8 & 1 & 21 & 13 & -5 & 13 \\
7 & 11 & 1 & 3 & -15 & 2 & -8 & 37 & -9 & 8 & $\mathbf{1 0 0}$ & 37 & -3 & 7 & 6 & 17 \\
8 & -2 & -13 & -8 & 5 & 1 & -9 & 41 & -1 & 1 & 37 & $\mathbf{1 0 0}$ & -2 & 10 & 22 & 29 \\
-24 & 3 & 30 & 31 & 49 & 34 & 64 & 25 & 17 & 21 & -3 & -2 & $\mathbf{1 0 0}$ & 14 & 6 & 24 \\
-7 & 2 & -5 & 9 & 12 & -5 & 14 & 10 & 7 & 13 & 7 & 10 & 14 & $\mathbf{1 0 0}$ & 10 & 9 \\
-2 & -5 & 0 & -4 & 1 & -2 & 16 & 11 & -1 & -5 & 6 & 22 & 6 & 10 & $\mathbf{1 0 0}$ & 50 \\
-28 & 7 & 12 & 11 & 16 & 2 & 31 & 31 & 11 & 13 & 17 & 29 & 24 & 9 & 50 & $\mathbf{1 0 0}$ \\
\hline
\end{tabular}

Table 3.2. Correlation matrix of monthly returns of the hedge funds. Rows and columns follow the same order as in Table 3.1

We showed in subsection 2.3 that the spectral risk measure of a Gaussian variable is an affine function of the mean and the standard deviation of this distribution. Thus optimizing under a risk constraint in a Gaussian framework is vacuous since it clearly leads to mean-variance efficient portfolios. We performed a Jarque-Bera test on our pool of hedge funds which shows that the marginal distributions are pretty far from Gaussian. Besides, using the test procedure developed in Malevergne and Sornette (2003), we show that the correlation structure of this 
pool of funds does not follow a Gaussian copula either. Indeed, the Kolmogorov-Smirnov procedure returns a distance of 0.34 for a sample of size 139; this corresponds to a P-value of less than $1 \%$.

We also provide risk measures for individual hedge funds (see Table 3.3), namely standard deviation (SD or StDev), lower semi-variance (LSV), Fischer risk measure (Fischer), absolute deviation to the mean (i.e. Denneberg absolute deviation or DAD) with $a=85 \%$, expected shortfall (ES) for $\alpha=10 \%$ and Wang-transform (W-T) for $\lambda=1.805$ which correspond to a reference quantile $\alpha=97 \%$ ).

As for the expected shortfall and Wang transform, we chose to calibrate them on the same level, using a Gaussian distribution as a benchmark. As a result, the expected shortfall at $10 \%$-quantile corresponds to the Wang transform with $\lambda=1.805$. Conversely, the choice of the parameter for Fischer's deviation to the average and Denneberg's deviation to the mean is irrelevant since these two risk measures are a linear combination of the expected return and a deviation term weighted by the parameter. The risk constraint is then completely endowed in the deviation term and the value of the parameter does not play any role - unless if it is equal to zero.

\begin{tabular}{|c|c|c|c|c|c|c|}
\hline & SD & LSV & $\operatorname{ES}(10 \%)$ & W-T $(\lambda=1.805)$ & Fischer R.M. & DAD \\
\hline HF 1 & $2,31 \%$ & $1,66 \%$ & $4,07 \%$ & $4,19 \%$ & $0,18 \%$ & $0,24 \%$ \\
\hline HF 2 & $4,31 \%$ & $3,04 \%$ & $7,28 \%$ & $6,98 \%$ & $0,91 \%$ & $0,89 \%$ \\
\hline HF 3 & $2,35 \%$ & $1,71 \%$ & $3,60 \%$ & $3,86 \%$ & $-0,33 \%$ & $-0,33 \%$ \\
\hline HF 4 & $2,16 \%$ & $1,51 \%$ & $2,52 \%$ & $3,19 \%$ & $-0,60 \%$ & $-0,65 \%$ \\
\hline HF 5 & $2,34 \%$ & $1,63 \%$ & $3,40 \%$ & $3,23 \%$ & $-0,11 \%$ & $-0,11 \%$ \\
\hline HF 6 & $0,71 \%$ & $0,54 \%$ & $0,68 \%$ & $0,66 \%$ & $-0,57 \%$ & $-0,53 \%$ \\
\hline HF 7 & $5,78 \%$ & $4,36 \%$ & $10,14 \%$ & $11,91 \%$ & $0,98 \%$ & $1,24 \%$ \\
\hline HF 8 & $1,86 \%$ & $1,25 \%$ & $2,17 \%$ & $2,27 \%$ & $-0,38 \%$ & $-0,42 \%$ \\
\hline HF 9 & $7,82 \%$ & $4,34 \%$ & $8,85 \%$ & $11,89 \%$ & $-0,24 \%$ & $-0,62 \%$ \\
\hline HF 10 & $1,00 \%$ & $0,68 \%$ & $1,02 \%$ & $1,25 \%$ & $-0,40 \%$ & $-0,38 \%$ \\
\hline HF 11 & $6,69 \%$ & $3,81 \%$ & $7,84 \%$ & $7,15 \%$ & $0,89 \%$ & $0,57 \%$ \\
\hline HF 12 & $4,17 \%$ & $2,68 \%$ & $4,73 \%$ & $4,89 \%$ & $-0,54 \%$ & $-0,70 \%$ \\
\hline HF 13 & $4,87 \%$ & $3,53 \%$ & $8,60 \%$ & $8,44 \%$ & $0,75 \%$ & $0,77 \%$ \\
\hline HF 14 & $5,58 \%$ & $3,66 \%$ & $7,87 \%$ & $8,32 \%$ & $1,04 \%$ & $0,84 \%$ \\
\hline HF 15 & $8,54 \%$ & $5,69 \%$ & $13,37 \%$ & $13,96 \%$ & $1,35 \%$ & $0,80 \%$ \\
\hline HF 16 & $8,40 \%$ & $5,56 \%$ & $12,56 \%$ & $13,22 \%$ & $1,12 \%$ & $0,61 \%$ \\
\hline
\end{tabular}

Table 3.3. Individual risk measurement of the hedge funds (monthly returns)

Now, in order to study the relative content of each risk measure, let us assess the rank correlations between the various risk measures for the 16 hedge funds of our dataset. The results are given in Table 3.4. Except for Denneberg and Fischer risk measures, we notice that the rankings of the 16 funds under the different risk measures are almost the same. We refer to Danielsson et al. (2006) for related results about the comparison of downside risk measures for heavy tailed distributions. 


\begin{tabular}{lrrrrrrr}
\hline & St Dev & LSV & ES & & W-T & FRM & \multicolumn{2}{c}{ DAD } \\
\hline \hline St Dev & 100 & 99 & 96 & 96 & 80 & 53 \\
LSV & 99 & 100 & 99 & 99 & 83 & 59 \\
ES & 96 & 99 & 100 & 100 & 83 & 60 \\
W-T & 96 & 99 & 100 & 100 & 83 & 60 \\
FRM & 80 & 83 & 83 & 83 & 100 & 89 \\
DAD & 53 & 59 & 60 & 60 & 89 & 100 \\
\hline
\end{tabular}

Table 3.4. Rank correlations among risk measures (same parameters as above), in \%

\subsection{Determining optimal portfolios}

The problem of portfolio optimization consists here in finding the allocation within the portfolio of 16 hedge funds which maximizes the expected return of the corresponding portfolio under some constraints such as no short-selling and some risk limit on the portfolio. Since the risk measures being considered here are coherent and thus convex, we can equivalently consider the dual problem of minimizing the risk measure under some expected return constraints.

Let us firstly detail the optimization problem. Each fund $i \in\{1, \ldots, 16\}$ is characterized by his historical returns $\left\{r_{1}, \ldots, r_{139}\right\}$. The return of the fund of funds corresponding to some portfolio allocation $a=\left(a_{1}, \ldots, a_{16}\right)$ is then given by $\pi(a)=a_{1} r^{1}+\ldots+a_{16} r^{16}$ and the corresponding historical returns are given as follows:

$$
\begin{array}{cc}
\text { Date } 1 & \pi(a)_{1}=a_{1} r_{1}^{1}+\ldots+a_{16} r_{1}^{16} \\
\vdots & \\
\text { Date } \boldsymbol{i} & \pi(a)_{i}=a_{1} r_{i}^{1}+\ldots+a_{16} r_{i}^{16} \\
\vdots &
\end{array}
$$$$
\text { Date } 139 \pi(a)_{139}=a_{1} r_{139}^{1}+\ldots+a_{16} r_{139}^{16}
$$

As an example, let us consider the case of a spectral risk measure with some spectrum $\phi$. The risk measure associated with portfolio allocation $a$ is performed by firstly sorting the historical returns: $\pi(a)_{1: 139} \leq \pi(a)_{2: 139} \leq \ldots \leq \pi(a)_{139: 139}$ and then by computing a weighted sum of these values, $-\sum_{i=1}^{139} \lambda_{i} \pi(a)_{i: 139}$, where $\lambda_{i}=\int_{(i-1) / 139}^{i / 139} \phi(p) d p$.

The optimal allocation problem can then we written as: $\min _{a}-\sum_{i=1}^{139} \lambda_{i} \pi(a)_{i: 139}$ under $a_{i} \geq 0$, $i=1, \ldots, 16$ (no short selling constraints), $a_{1}+\cdots+a_{16}=1$ (budget constraint) and $\frac{1}{139} \sum_{i=1}^{139} \pi(a)_{i}=\bar{r}$ (expected return constraint). 
There exist various efficient numerical techniques based on linear programming to solve this problem. Rockafellar and Uryasev (2000) pioneered these techniques in the case of the expected shortfall. Acerbi and Simonetti (2002) and Bassett et al. (2004) further extended the approach to the case of spectral and distortion risk measures. As for variance-based measures, we can rely on quadratic optimization methods as shown in Konno et al. (2002).

Figures 3.5, 3.6 and 3.7 represent the optimal portfolios for variance-based risk measures (lower semi-variance and standard deviation), moment-based measures and some spectral risk measures. We show how the optimal allocation depends upon the expected return objective.

- Efficient allocations for variance-based risk measures
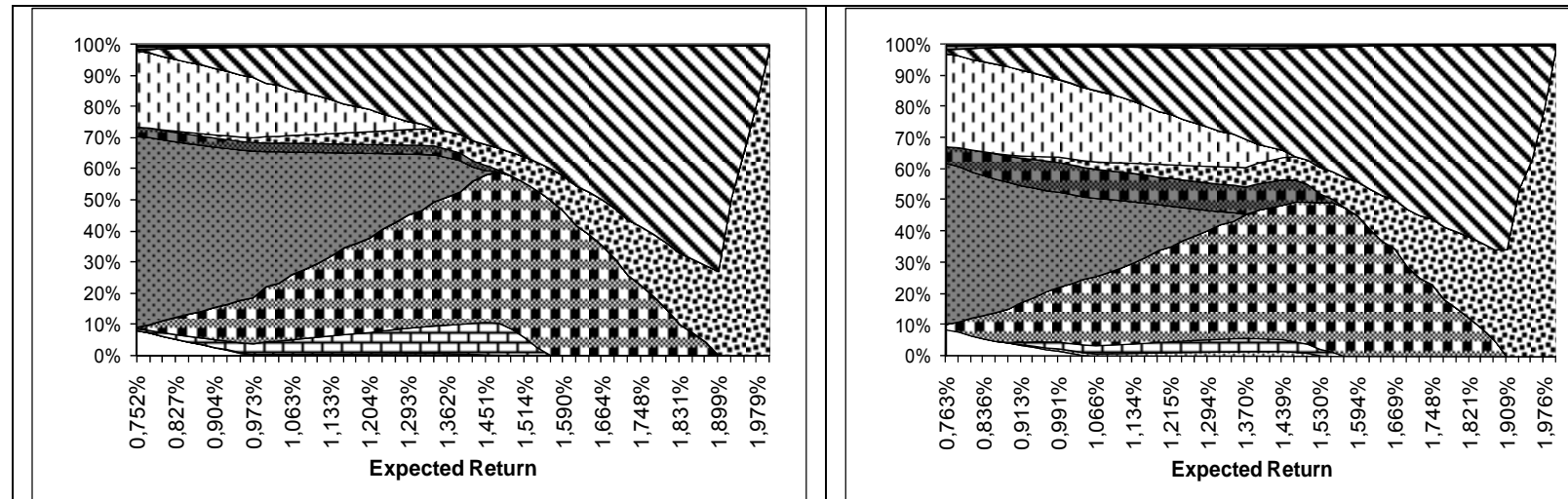

Fig 3-5: Efficient allocations for standard deviation (left) and lower semi-variance (right)

- Case of deviation-based measures

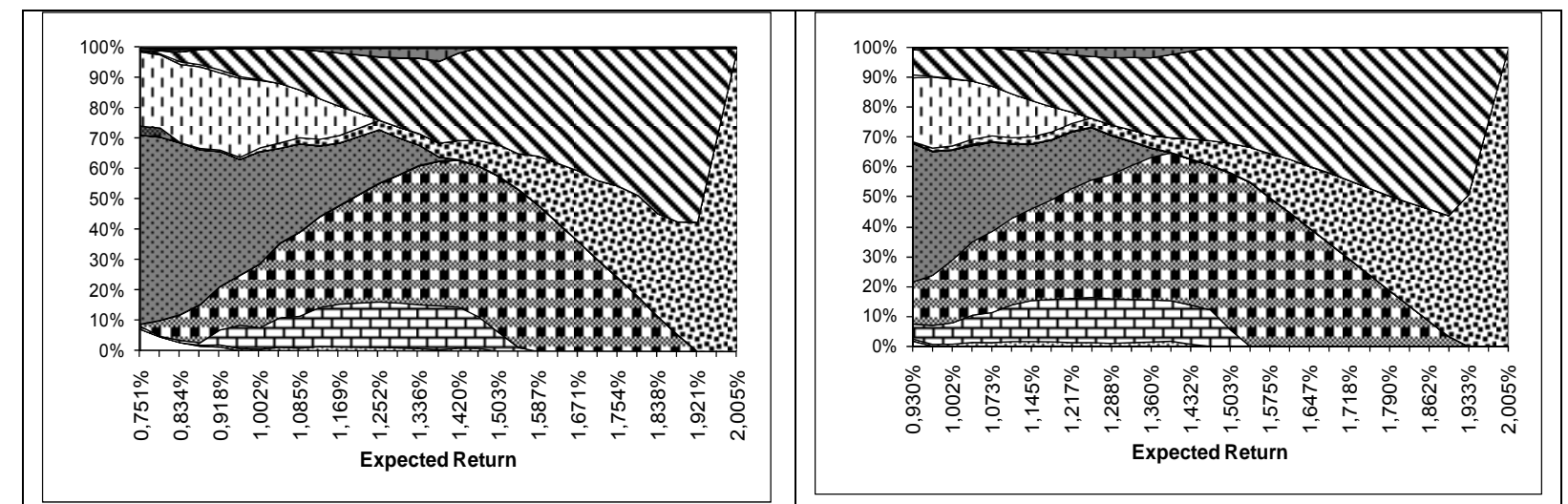

Fig 3-6: Efficient allocations for Fischer measure ( $a=85 \%)($ left) and Denneberg absolute deviation $($ right $)(a=85 \%)$

- Spectral risk measures 


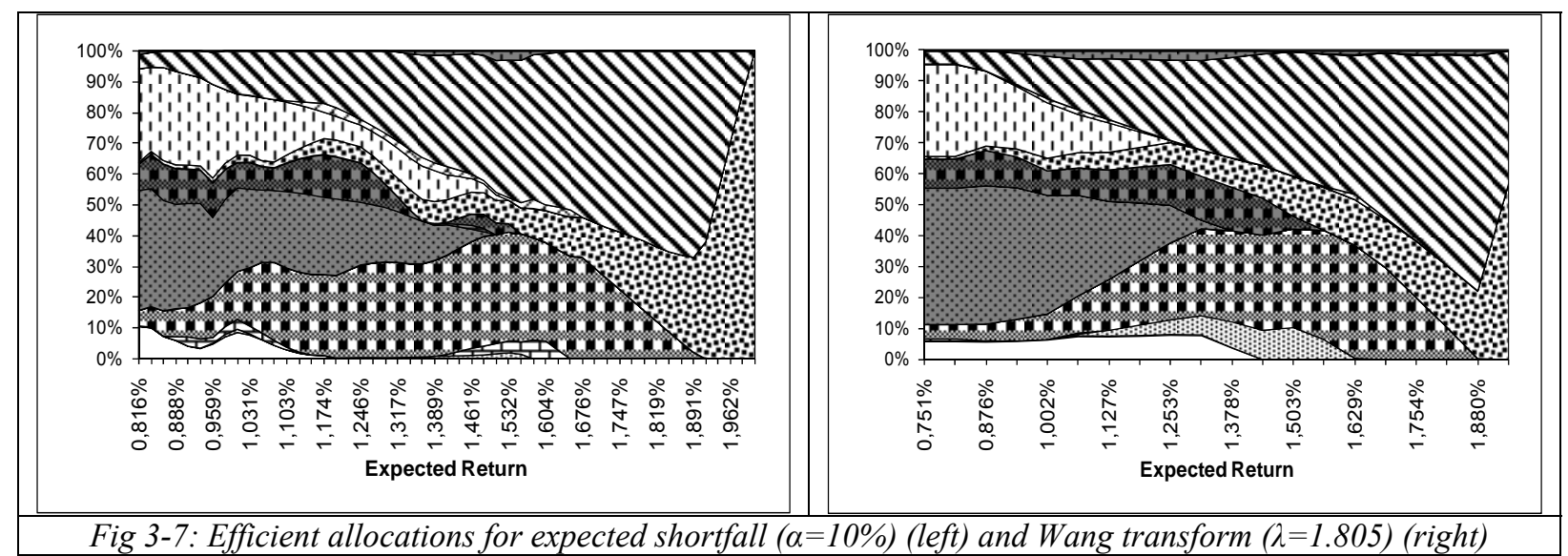

\section{Caption}

\begin{tabular}{|c|c|}
\hline$\square$ AXA Rosenberg Market Neutral Strategy LP & F Discovery MasterF und Ltd \\
\hline B Aetos Corporation & ⿴囗Bennett Restructuring F und LP \\
\hline$\square$ Calamos Convertible Hedge Fund LP & ⿴囗S Sage Capital Limited Partnership \\
\hline G Genesis Emerging Markets Fund Ltd & 口RXR Secured Participating Note \\
\hline E Arrowemith F und Ltd & ๑ Blue Rock Capital Fund LP \\
\hline Gean Witter Corner stone Fund N LP & D GAMut Investments Inc \\
\hline$\square$ Aquila International $F$ und Ltd & ๑ Bay Capital Management \\
\hline G Blenheim Irvestments LP (Composite) & $\mathbf{\Delta R e d ~ O a k ~ C o m m o d i t y ~ A d v i s o r s ~ I n c ~}$ \\
\hline
\end{tabular}

At first sight, there are only small differences as we skip from one risk measure to another. Some other studies have recently investigated the use of alternative risk measures for portfolio management. For example, Krokhmal et al. (2002) performed hedge fund portfolio optimization under different risk measures: CVaR, conditional drawdown at risk, mean absolute deviation and maximum loss, showing that the resulting efficient frontiers are close. Chabaane et al. (2006) compared VaR optimal portfolios computed under different VaR estimators and expected shortfall or semi-variance optimal portfolios. Their study shows some similarity between the various optimal allocations as well.

\subsection{Analysing efficient portfolios}

We further investigate this proximity issue by firstly quantifying the proximity between optimal portfolios. For this purpose, we chose some level of expected return and we determined the corresponding optimal portfolio for each risk measure. For our study we chose the level of return corresponding to the portfolio equally weighted in each hedge fund, noticing that this return $-1.04 \%$ - is located in a region where efficient portfolios are significantly diversified, as we see in the previous figures. This leads to six optimal funds of hedge funds each of them being associated with a particular risk constraint. In Table 3.8, we report their corresponding risk characteristics and we notice that they almost do not change when switching from one portfolio to another.

\begin{tabular}{lrrrrrr}
\hline & \multicolumn{7}{c}{ Efficient Portfolios } \\
\cline { 2 - 7 } & St Dev & LSV & ES & Wang & DAD & FRM \\
\hline \hline Expected return & $1,04 \%$ & $1,04 \%$ & $1,04 \%$ & $1,04 \%$ & $1,04 \%$ & $1,04 \%$ \\
Minimum return & $-1,72 \%$ & $-1,48 \%$ & $-1,38 \%$ & $-0,64 \%$ & $-2,14 \%$ & $-2,12 \%$
\end{tabular}




\begin{tabular}{lrrrrrr} 
Maximum return & $3,81 \%$ & $3,86 \%$ & $3,86 \%$ & $4,27 \%$ & $4,33 \%$ & $4,32 \%$ \\
Standard deviation & $0,89 \%$ & $0,92 \%$ & $0,92 \%$ & $1,01 \%$ & $0,91 \%$ & $0,91 \%$ \\
Lower semi-variance & $0,62 \%$ & $0,60 \%$ & $0,61 \%$ & $0,64 \%$ & $0,64 \%$ & $0,64 \%$ \\
Expected shortfall & $0,30 \%$ & $0,24 \%$ & $0,21 \%$ & $0,34 \%$ & $0,34 \%$ & $0,35 \%$ \\
Wang transform & $0,56 \%$ & $0,48 \%$ & $0,45 \%$ & $0,29 \%$ & $0,72 \%$ & $0,71 \%$ \\
Denneberg absolute deviation & $-0,66 \%$ & $-0,70 \%$ & $-0,66 \%$ & $-0,69 \%$ & $-0,72 \%$ & $-0,71 \%$ \\
Fischer risk measure & $-0,71 \%$ & $-0,70 \%$ & $-0,68 \%$ & $-0,65 \%$ & $-0,72 \%$ & $-0,72 \%$ \\
\hline
\end{tabular}

Table 3.8. Some characteristics of the six efficient portfolios (monthly returns)

As mentioned in Hahn et al. (2002) or Sharma (2004), the proximity between efficient portfolios can be well captured by computing the rank correlation between their historical returns. Thus, the rank correlations between the 6 efficient portfolios mentioned above are also very explicit (Table 3.9) since all of them are located beyond $80 \%$. We also led a principal component analysis on the returns of these 6 optimal portfolios, showing that the first factor explains more than $90 \%$ of the total dispersion of our 6 portfolios. These results contribute to show some kind of robustness of optimal allocations with respect to the choice of the risk measure. Therefore, our study confirms and extends the results of Krokhmal et al. (2002), Chabaane et al. (2006) to a wider range of spectral and deviation-based risk measures.

\begin{tabular}{|c|c|c|c|c|c|c|}
\hline & St Dev & LSV & ES & Wang & DAD & FRM \\
\hline St Dev & $100 \%$ & $98 \%$ & $97 \%$ & $89 \%$ & $97 \%$ & $97 \%$ \\
\hline LSV & $98 \%$ & $100 \%$ & $99 \%$ & $92 \%$ & $94 \%$ & $94 \%$ \\
\hline ES & $97 \%$ & $99 \%$ & $100 \%$ & $92 \%$ & $93 \%$ & $93 \%$ \\
\hline Wang & $89 \%$ & $92 \%$ & $92 \%$ & $100 \%$ & $81 \%$ & $81 \%$ \\
\hline DAD & $97 \%$ & $94 \%$ & $93 \%$ & $81 \%$ & $100 \%$ & $100 \%$ \\
\hline FRM & $97 \%$ & $94 \%$ & $93 \%$ & $81 \%$ & $100 \%$ & $100 \%$ \\
\hline
\end{tabular}

Table 3.9. Rank correlations between efficient portfolios (same portfolios as in Table 3.8)

To further investigate the robustness of portfolio selection with respect to the choice of risk measure, we computed optimal portfolio allocations as a function of the threshold of the expected shortfall. This was achieved for 7 values of the threshold $-5 \%, 10 \%, 15 \%, 20 \%$, $30 \%, 40 \%$ - and an expected return of $1.04 \%$. The same study as above leads to over $80 \%$ rank correlations and a PCA shows that more than $95 \%$ of the total dispersion is related to a unique factor This confirms the proximity between optimal allocations with respect to the choice of the measure since, as mentioned in subsection 2.5, any spectral risk measure can be expressed as a weighted average of expected shortfalls at different probability thresholds.

However, when going to extreme attitudes towards risk, we observe new kinds of optimal investments. As we did for other risk measures, we represent in Figure 3.10 the optimal allocations for the risk measure representing the worst case scenario with respect to the expected return objective. This latter risk measure corresponds to the lowest quantile of the distribution and is equal, in our framework, to an expected shortfall at a level $\alpha \leq 1 / n$. 


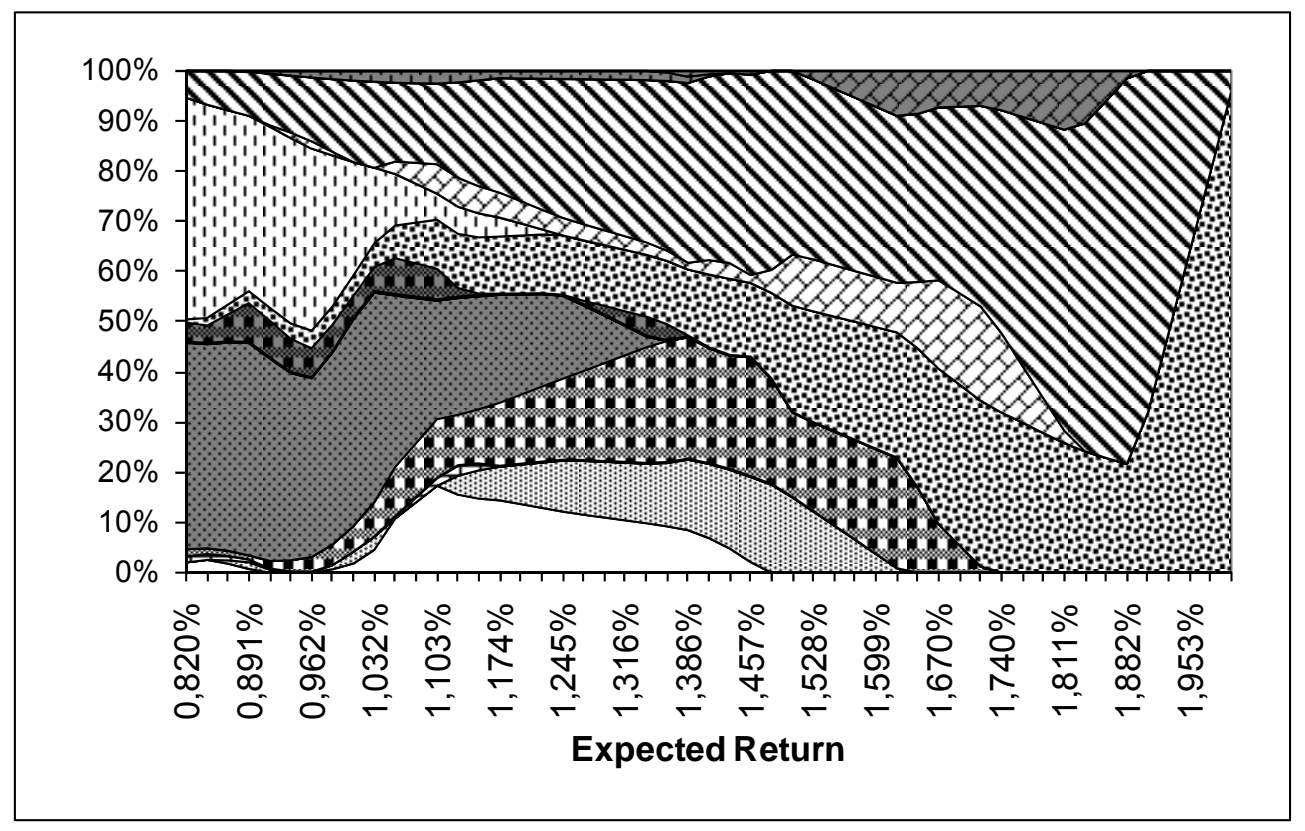

Fig. 3.10. Efficient Allocations for Worst Case risk measure

Comparing with the same graph for the expected shortfall at a 10\% level (Fig.3.7), some funds such as HF1 or HF2 appear in a more important proportion in the intermediate range of expected returns $(1.1 \%-1.6 \%)$. The presence of such funds is likely to be related to diversification effects decreasing the global risk of the portfolio. Thus, we studied more specifically the diversification effect by computing the diversification ratio defined as $D R(a)=\left(\sum_{i=1}^{16} a_{i}^{2}\right)^{-1}$. We show the evolution of this ratio in Figure 3.11. The higher $\operatorname{DR}(a)$, the more diversified a portfolio will be. We see that the statement that more pessimistic investors tend to hold more diversified portfolios is somehow true but does not explain everything.

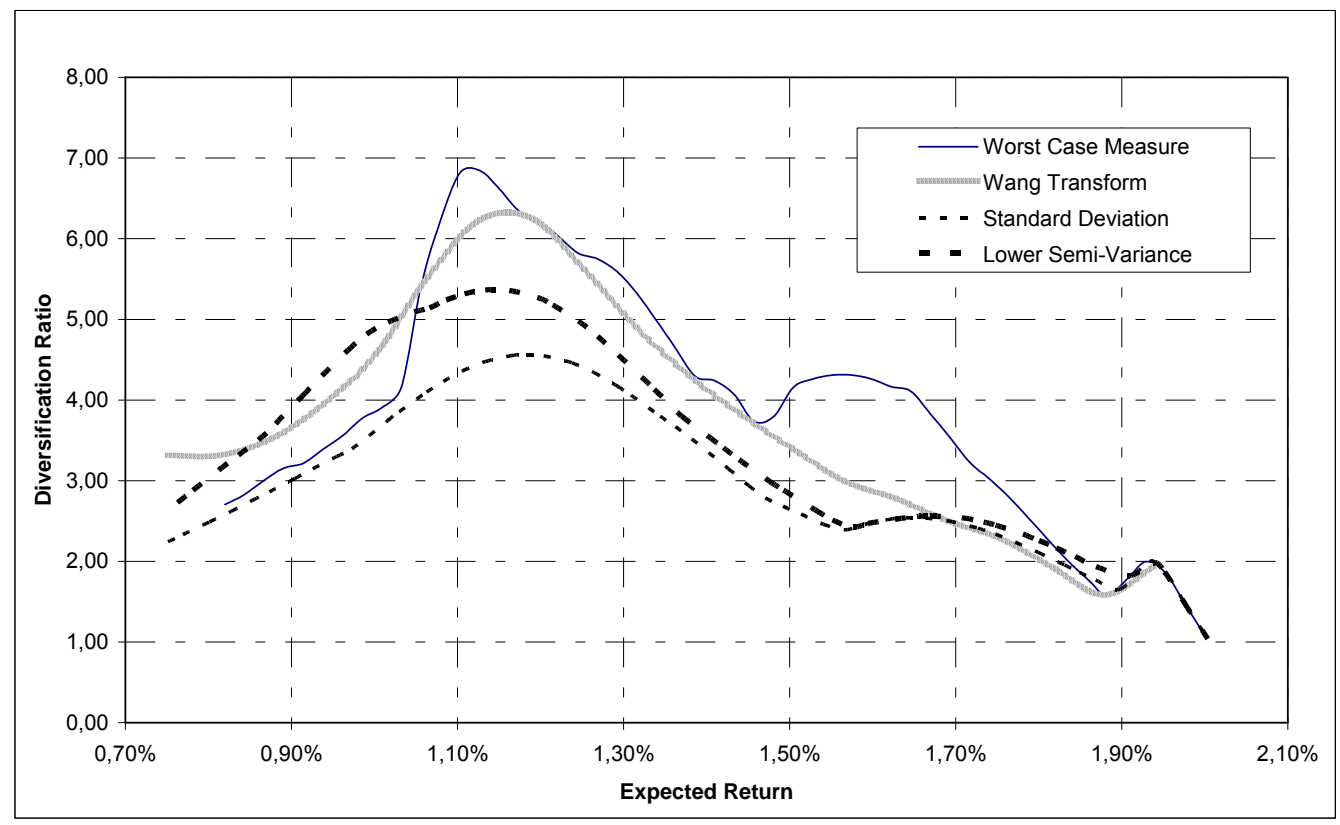

Fig. 3.11. Diversification ratio of efficient portfolios for several risk measures varying expected return 
For extremely demanding risk constraints - corresponding to the left part of the diversification ratio curves - optimal portfolios are concentrated on fewer funds, those with the « less catastrophic » risk characteristics. To illustrate it, we focus on HF 6, the fund whose lowest quantile is the highest among the funds of the pool. As Table 3.12 shows, its proportion in optimal portfolios goes higher as the risk constraint hardens.

\begin{tabular}{lrrrrrrr}
\hline Expected Return & $0.80 \%$ & $0.90 \%$ & $1.00 \%$ & $1.10 \%$ & $1.20 \%$ & $1.30 \%$ & $1.40 \%$ \\
\hline \hline Standard Deviation & $58.33 \%$ & $51.87 \%$ & $44.92 \%$ & $36.29 \%$ & $27.66 \%$ & $19.03 \%$ & $7.87 \%$ \\
Lower Semi-Variance & $48.19 \%$ & $38.80 \%$ & $29.99 \%$ & $22.45 \%$ & $14.40 \%$ & $6.02 \%$ & $0.02 \%$ \\
Worst Case Measure & $41.62 \%$ & $41.74 \%$ & $40.27 \%$ & $24.42 \%$ & $19.89 \%$ & $8.28 \%$ & $0.00 \%$ \\
Wang Transform & $44.25 \%$ & $44.01 \%$ & $38.58 \%$ & $28.48 \%$ & $17.73 \%$ & $5.08 \%$ & $0.00 \%$ \\
\hline
\end{tabular}

Table 3.12. Proportion of HF 6 in efficient portfolios when varying expected return

The latter results show the influence of two opposite effects. On one hand, as mentioned above, an increased risk constraint should lead to more diversified portfolios. On the other hand, diversification effects may be counterbalanced in case of positive tail dependence. For instance, in the specific case where extreme negative returns would be perfectly correlated, the optimal portfolio with respect to the worst case risk measure would consist in a full allocation in the fund with the highest worst return, here HF 6.

\section{Conclusion}

Various classes of risk measures - spectral or distortion risk measures, moment based risk measures - can be used in the framework of portfolio optimization. We firstly reviewed some theoretical properties of such risk measures and discussed the use of the empirical measure.

Thanks to linear or quadratic programming techniques, the problem of finding optimal portfolio allocations under a risk constraint can be efficiently solved. As the panorama of risk measures has considerably widened, there remains, for the asset manager, the question of the choice of the measure. Our case study based on a dataset of individual hedge funds shows some robustness of the optimal allocations with respect to the choice of the measure and confirms previous studies. However, as we head for more extreme risk criteria like the worst case measure, we obtain slightly different optimal profiles. The optimal allocations ensue from two opposite effects. Diversification tends to be magnified as the risk constraint sharpens but due to possible dependence effects of extreme returns, the rational investor might concentrate its investments on the less risky funds.

\section{Acknowledgements}

We thank Julien Salomon for his significant contribution to the development of optimization tools and ideas within this paper and Mehdi Mlaiki for computational assistance. We also thank Steve Kou, Yannick Malevergne, Olivier Scaillet, Françoise Turpin, Shaun Wang and the participants of the French Finance annual meeting and of the Universities of Lyon and of Lausanne joint actuarial seminar for useful comments and discussions. The usual disclaimer applies.

\section{Appendix A: Scenario analysis and spectral risk measures in a discrete framework}


In this Appendix, we successively establish a representation of the expected shortfall and spectral risk measures through scenario analysis, as developed in Artzner et al. (1999) or Heyde et al. (2006).

Proposition A.1. (Scenario analysis and expected shortfall at level $\alpha$ ) For some permutation $\sigma$ of the set $\{1, \ldots, n\}$, we define $P_{\alpha}^{\sigma}$ as the probability measure such that: $\left\{\begin{array}{l}\forall 1 \leq k \leq\lceil n \alpha\rceil-1, P_{\alpha}^{\sigma}\left(\omega_{\sigma(k)}\right)=\frac{1}{n \alpha}, \\ P_{\alpha}^{\sigma}\left(\omega_{\sigma(\lceil n\rceil))}\right)=\frac{n \alpha-\lceil n \alpha\rceil+1}{n \alpha}, \\ \forall(\lceil n \alpha\rceil+1) \leq k \leq n, P_{\alpha}^{\sigma}\left(\omega_{\sigma(k)}\right)=0 .\end{array}\right.$

Then we set $\Pi_{\alpha}=\left\{P_{\alpha}^{\sigma}, \sigma \in \mathrm{S}_{n}\right\}$ where $\mathrm{S}_{n}$ stands for the set of the permutations of $\{1, \ldots, n\}$. With these notations, we have the following core representation of the expected shortfall at level $\alpha$ :

$$
\operatorname{ES}_{\alpha}(X)=\sup \left\{-E^{P}[X], P \in \Pi_{\alpha}\right\}
$$

Moreover, for any random variable $X$, the upper bound is reached for any permutation $\sigma_{X}$ fulfilling $X\left(\omega_{\sigma_{X}(1)}\right) \leq X\left(\omega_{\sigma_{X}(2)}\right) \leq \ldots \leq X\left(\omega_{\sigma_{X}(n)}\right)$.

Proof of Proposition A.1. Under our assumption of the space $\Omega$ being finite, the portfolio return $X$ takes a finite number of values which can be ordered $x_{1: n} \leq x_{2: n} \leq \ldots \leq x_{n: n}$. We define the corresponding events $\left(\omega_{1: n} ; \ldots ; \omega_{n: n}\right)$ such that $X\left(\omega_{1: n}\right)=x_{1: n} ; \ldots ; X\left(\omega_{n: n}\right)=x_{n: n}$. Such a permutation of the events corresponds to $\sigma_{X}$ as defined in Proposition B.1, we call it an ordering permutation.

Then we have $\operatorname{ES}_{\alpha}(X)=-\frac{1}{n \alpha}\left(\sum_{i=1}^{\lceil n \alpha\rceil-1} x_{i: n}+(n \alpha-\lceil n \alpha\rceil+1) x_{\lceil n \alpha\rceil: n}\right)=-E^{P_{\alpha}^{\sigma(X)}}[X]$, according to the notations we adopted.

In the proposition we state that computing the expected shortfall of $X$ coincides with the maximal value of $-E^{P_{\alpha}^{\sigma}}[X]$ over all permutations $\sigma$ and that this maximum is attained for any ordering permutation $\sigma_{X}$. Let us show this point.

For any pair of indexes $(i, j)$ such that $1 \leq i \leq j \leq n$ we have $P_{\alpha}\left(\omega_{i}\right) x_{j: n}+P_{\alpha}\left(\omega_{j}\right) x_{i: n} \geq P_{\alpha}\left(\omega_{i}\right) x_{i: n}+P_{\alpha}\left(\omega_{j}\right) x_{j: n}$ since $P_{\alpha}\left(\omega_{1}\right) \geq \ldots \geq P_{\alpha}\left(\omega_{n}\right)$. Since, according to our notations, we have:

$$
E^{P_{\alpha}^{\sigma}}[X]=\sum_{k=1}^{n} P_{\alpha}\left(\omega_{k}\right) x_{\sigma(k)},
$$

this means that any time one switches two indexes in the ordering permutation, he obtains an expected value which is superior to $E^{P_{\alpha}^{\sigma(X)}}[X]$. More generally speaking, applying any permuting cycle to $\sigma_{X}$ leads to the same result. Since any permutation can be written as a 
composition of independent permuting cycles, this shows that $\sigma_{X}$ is a permutation minimizing the quantity $E^{P_{\alpha}^{\sigma}}[X]$.

By this way we obtain one of the possible set of scenarios characterizing the expected shortfall:

$$
\Pi_{\alpha}=\left\{P_{\alpha}^{\sigma} \mid \sigma \in \mathrm{S}_{n}\right\}
$$

Moreover, all these probability measures are absolutely continuous with respect to the historical probability measure.

A similar result can be established for spectral risk measures:

Proposition A.2. (Scenario analysis and spectral risk measures) Let $M_{\phi}$ be a spectral risk measure characterized by its spectrum $\phi$. For some permutation $\sigma$ of the set $\{1, \ldots, n\}$, we define the probability measure $P_{\phi}^{\sigma}$ by $\forall 1 \leq k \leq n, P_{\phi}^{\sigma}\left(\omega_{\sigma(k)}\right)=\int_{(k-1) / n}^{k / n} \phi(p) d p$ and we set $\Pi_{\phi}=\left\{P_{\phi}^{\sigma}, \sigma \in \mathrm{S}_{n}\right\}$ where $\mathrm{S}_{n}$ still stands for the set of the permutations of $\{1, \ldots, n\}$.

With these notations, we have the following representation of $M_{\phi}$ :

$$
M_{\phi}(X)=\sup \left\{-E^{P}[X], P \in \Pi_{\phi}\right\}
$$

Moreover, for any fixed random variable $X$, the superior bound is attained for any ordering permutation $\sigma_{X}$ defined by $X\left(\omega_{\sigma_{X}(1)}\right) \leq X\left(\omega_{\sigma_{X}(2)}\right) \leq \ldots \leq X\left(\omega_{\sigma_{X}(n)}\right)$.

Proof of Proposition A.2. This proof is a mere generalisation of the proof concerning the expected shortfall to spectral risk measures. In the notations, the probability measure $P_{\alpha}$ is replaced by the measure $P_{\phi}$ characterized by the values $P_{\phi}=\left(\lambda_{1}, \ldots, \lambda_{n}\right)$ where $\lambda_{k}=\int_{(k-1) / n}^{k / n} \phi(p) d p$. Moreover, we notice that $\lambda_{1} \geq \ldots \geq \lambda_{n} \geq 0$ and $\sum_{i=1}^{n} \lambda_{i}=1$ due to the assumptions made on the spectrum $\phi$.

According to these notations and keeping the concept of the ordering permutation $\sigma_{X}$, we have:

$$
M_{\phi}(X)=-\sum_{k=1}^{n} \lambda_{k} x_{k: n}=-E^{P_{\phi}}\left[X_{\sigma(X)}\right] .
$$

Since the weights of the measure $P_{\phi}$ are non-increasingly ordered, the idea using permuting cycles in the case of the expected shortfall can be used the same way for spectral risk measures (see proof of Proposition A.1). Thus, as in the case of the expected shortfall, the spectral risk measure $M_{\phi}$ can be set under the following form:

$$
M_{\phi}(X)=\max \left\{-E^{P_{\phi}^{\sigma}}[X] \mid \sigma \in \mathrm{S}_{n}\right\}
$$


where for any $\sigma \in \mathrm{S}_{n}, P_{\phi}^{\sigma}$ is the probability measure obtained by permuting the values of $P_{\phi}$ according to $\sigma$. Similarly, the maximum value is attained for the ordering permutation $\sigma_{X}$.

As a consequence, we can derive a closed form for the set of scenarios characterizing a spectral risk measure:

$$
\Pi_{\phi}=\left\{P_{\phi}^{\sigma} \mid \sigma \in \mathrm{S}_{n}\right\}
$$

\section{Appendix B: Proof of the consistency of ordering risks with spectral risk measures and second order stochastic dominance}

In this Appendix, we focus on the following result:

\section{Proposition B.1. (Consistency of spectral risk measures ordering with second order stochastic dominance):}

$$
X \underset{S O S D}{\leq} Y \Leftrightarrow \text { For any probability threshold } \alpha \text { we have } \operatorname{ES}_{\alpha}(X) \geq \operatorname{ES}_{\alpha}(Y)
$$

and

$$
X \underset{S O S D}{\leq} Y \Leftrightarrow \text { For any spectral risk measure } M_{\phi} \text { we have } M_{\phi}(X) \geq M_{\phi}(Y) \text {. }
$$

Proof of Proposition B.1. Since any spectral risk measure can be expressed as a positively weighted average of expected shortfalls, and since the expected shortfalls belong to the class of spectral risk measures, establishing one of these equivalences gives the other one.

Then we focus on the first one and to achieve the proof, we refer to the method developed in Denuit et al. (2006) with adaptations. An interesting proof of this result can also be found in Leitner (2005). We recall that standard microeconomic theory (see Denuit et al. (2006) for example) allows to equivalently write the SOSD order using the distribution functions of the returns:

$$
X \underset{S O S D}{\leq} Y \Leftrightarrow \forall t \in \mathbb{R}, \int_{t}^{+\infty}\left(1-F_{X}(u)\right) d u \leq \int_{t}^{+\infty}\left(1-F_{Y}(u)\right) d u .
$$

Proof of $\Rightarrow$. We suppose that $X \underset{S O S D}{\leq} Y$. We refer to the characterization of SOSD with utility functions considering the case of stop-loss functions $t \mapsto-(a-t)^{+}$defined for every $a \in \mathbb{R}$. Then the SOSD-domination of $Y$ on $X$ implies that for every $a \in \mathbb{R}$ we have:

$$
E^{P}\left[(a-X)^{+}\right] \geq E^{P}\left[(a-Y)^{+}\right]
$$

Let $p \in(0,1]$ be some probability level. We know (see for example Rockafellar and Uryasev, (2002) or Acerbi and Tasche (2002)) that the expected shortfall of $X$ at level $p$ is the 
minimum value of $\left\{-b+\frac{1}{p} E\left[(b-X)^{+}\right]\right\}$upon $b \in \mathbb{R}$. We also know that the minimum is attained for $b=-\operatorname{VaR}_{p}(X)=F_{X}^{-1}(p)$.

Thus, the end of the proof is straightforward:

$$
\begin{aligned}
\operatorname{ES}_{p}[Y] & =\inf _{b \in \mathbb{R}}\left\{-b+\frac{1}{p} E\left[(b-Y)^{+}\right]\right\} \leq \operatorname{VaR}_{p}(X)+\frac{1}{p} E\left[\left(-\operatorname{VaR}_{p}(X)-Y\right)^{+}\right] \\
& \leq \operatorname{VaR}_{p}(X)+\frac{1}{p} E\left[\left(-\operatorname{VaR}_{p}(X)-X\right)^{+}\right]=\operatorname{ES}_{p}[X]
\end{aligned}
$$

Proof of $\Leftarrow$. Here we require the theory of the integration of non-increasing and non-negative functions and their pseudo inverse developed for example in Denneberg (1994).

We suppose that $\operatorname{ES}_{\alpha}(X) \geq \operatorname{ES}_{\alpha}(Y)$ for every probability threshold $\alpha \in(0,1]$ and we will show that $\forall t \in \mathbb{R}, \int_{t}^{+\infty}\left(1-F_{Y}(u)\right) d u \geq \int_{t}^{+\infty}\left(1-F_{X}(u)\right) d u, \quad$ or $\quad$ equivalently $\forall t \in \mathbb{R}, \int_{t}^{+\infty} F_{X}(u) d u \geq \int_{t}^{+\infty} F_{Y}(u) d u$. First, we establish the following Lemma:

Lemma B.2. For every $x \in \mathbb{R}$, we have:

$$
\int_{x}^{+\infty}(1-F(u)) d u=\int_{F(x)}^{1} F^{-1}(p) d p-x(1-F(x)),
$$

where $F$ stands for the cumulated density function of either $X$ or $Y$ and $F^{-1}$ the corresponding quantile function.

Proof of Lemma B.2. We consider $x \in \mathbb{R}$ and the function

$$
\bar{F}_{X}^{x}: u \in \mathbb{R}^{+} \rightarrow 1-F_{X}(u+x) \in \mathbb{R}^{+} .
$$

This function is non-negative and non-increasing. Thus we can define

$$
\left(\bar{F}_{X}^{x}\right)^{-1}: p \in \mathbb{R}^{+} \rightarrow 0 \vee \sup \left\{u \mid \bar{F}_{X}^{x}(u)>p\right\}
$$

as the pseudo-inverse of $\bar{F}_{X}^{x}$ in the sense of Denneberg (1994). Here $a \vee b$ stands for $\max (a, b)$.

According to Denneberg's results, we have

$$
\int_{0}^{+\infty} \bar{F}_{X}^{x}(u) d u=\int_{0}^{+\infty}\left(\bar{F}_{X}^{x}\right)^{-1}(p) d p
$$

Further computation of $\left(\bar{F}_{X}^{x}\right)^{-1}$ gives the following: 


$$
\begin{aligned}
\left(\bar{F}_{X}^{x}\right)^{-1}( & p)=0 \vee \sup \left\{u \mid \bar{F}_{X}^{x}(u)>p\right\} \\
= & 0 \vee \sup \left\{u \mid 1-F_{X}(u+x)>p\right\} \\
= & 0 \vee \sup \left\{u \mid F_{X}(u+x)<1-p\right\} \\
= & 0 \vee\left[-x+\sup \left\{u \mid F_{X}(u)<1-p\right\}\right]
\end{aligned}
$$

Since $\sup \left\{u \mid F_{X}(u)<1-p\right\}=\inf \left\{u \mid F_{X}(u) \geq 1-p\right\}=F_{X}^{-1}(1-p)$ then:

$$
\left(\bar{F}_{X}^{x}\right)^{-1}(p)=0 \vee\left[F_{X}^{-1}(1-p)-x\right] \text {. }
$$

Coming back to equality (C.3), we compute separately each of the two terms:

$$
\int_{0}^{+\infty} \bar{F}_{X}^{x}(u) d u=\int_{0}^{+\infty}\left(1-F_{X}(u+x)\right) d u=\int_{x}^{+\infty}\left(1-F_{X}(u)\right) d u
$$

in the second one, we firstly notice that the 0 term is the greater in the maximum form of $\left(\bar{F}_{X}^{x}\right)^{-1}$ when $1-p>F_{X}(x)$, or equivalently $p<1-F_{X}(x)$. This leads to:

$$
\int_{0}^{+\infty}\left(\bar{F}_{X}^{x}\right)^{-1}(u) d u=\int_{0}^{1-F_{X}(x)}\left[F_{X}^{-1}(1-p)-x\right] d p=-x\left(1-F_{X}(x)\right)+\int_{F_{X}(x)}^{1} F_{X}^{-1}(p) d p .
$$

Eventually, we get:

$$
\int_{x}^{+\infty}\left(1-F_{X}(u)\right) d u=-x\left(1-F_{X}(x)\right)+\int_{F_{X}(x)}^{1} F_{X}^{-1}(p) d p
$$

this achieves the proof of Lemma B.2.

Let $X$ and $Y$ be two random variables verifying $\operatorname{ES}_{\alpha}(X) \geq \operatorname{ES}_{\alpha}(Y)$ for every probability threshold $\alpha$. This means that for any $\alpha \in(0,1], \int_{1-\alpha}^{1} F_{X}^{-1}(p) d p \geq \int_{1-\alpha}^{1} F_{Y}^{-1}(p) d p$. Let $x \in \mathbb{R}$.

First, we assess the case where $F_{X}(x) \leq F_{Y}(x)$. According to Lemma B.2, we have:

$$
\int_{x}^{+\infty}\left[F_{X}(u)-F_{Y}(u)\right] d u=\int_{F_{X}(x)}^{1}\left[F_{Y}^{-1}(p)-F_{X}^{-1}(p)\right] d p+\int_{F_{X}(x)}^{F_{Y}(x)}\left[x-F_{Y}^{-1}(p)\right] d p
$$

The first term is non-negative and the second one either, since

$$
\forall p \in\left[F_{X}(x), F_{Y}(x)\right], F_{Y}^{-1}(p) \leq F_{Y}^{-1}\left(F_{Y}(x)\right)=\inf \left\{u \mid F_{Y}(u) \geq F_{Y}(x)\right\} \leq x,
$$

giving $\int_{x}^{+\infty}\left[F_{X}(u)-F_{Y}(u)\right] d u \geq 0$.

Now, let us consider the case where $F_{X}(x)>F_{Y}(x)$ - or equivalently $1-F_{Y}(x)>1-F_{X}(x)$. First, if we suppose that $F_{X}(t)>F_{Y}(t)$ for every $t \geq x$, then the result is obvious. Therefore we suppose that there exists $x_{0} \geq x$ such that $F_{X}\left(x_{0}\right) \leq F_{Y}\left(x_{0}\right)$. Thus, we consider

$$
\bar{x}=\inf \left\{t \geq x \mid F_{X}(t) \leq F_{Y}(t)\right\} \text {. }
$$


We know that $\forall t \in[x, \bar{x}), F_{X}(t) \geq F_{Y}(t)$, giving $\int_{x}^{\bar{x}} F_{X}(t) d t \geq \int_{x}^{\bar{x}} F_{Y}(t) d t$.

Besides, by right-continuity of the cumulated density functions, we have $F_{X}(\bar{x}) \leq F_{Y}(\bar{x})$. Using equation (B.4) with $x=\bar{x}$ gives $\int_{\bar{x}}^{+\infty} F_{X}(t) d t \geq \int_{\bar{x}}^{+\infty} F_{Y}(t) d t$, leading to the desired inequality and achieving the proof of Proposition B.1.

\section{Appendix C: Spectral Risk Measures as Weighted Averages of Expected Shortfalls.}

In this Appendix, we establish the following proposition:

Proposition C.1. Any spectral risk measure can be expressed as a positively weighted average of Expected Shortfalls. In other words, given a spectral risk measure $M_{\phi}$, there exists a probability measure $v$ on $[0,1]$ such that

$$
M_{\phi}(X)=\int_{0}^{1} \operatorname{ES}_{\alpha}(X) d v(\alpha) .
$$

Proof of Proposition C.1. To show this, let us denote by $\mu$, the positive measure such that $\mu([a, b))=\phi(a)-\phi(b)$, for $0<a<b \leq 1$.

Thus, $\phi(p)=\phi(1)+\int 1_{[p, 1)}(u) d \mu(u)$. Since $\quad E^{P}[X]=\int_{0}^{1} F_{X}^{-1}(p) d p=-\operatorname{ES}_{1}(X) \quad$ and $u$ using Fubini's theorem, $M_{\phi}(X)=\phi(1) \mathrm{ES}_{1}(X)+\int_{0}^{1} \alpha \mathrm{ES}_{\alpha}(X) d \mu(\alpha)$. Let us eventually consider the measure $v$ defined by $v(B)=\phi(1) \delta_{1}(B)+\int_{B} \alpha d \mu(\alpha)$ for any Borel subset of $[0,1], B$. Then,

$$
M_{\phi}(X)=\int_{0}^{1} \operatorname{ES}_{\alpha}(X) d v(\alpha) .
$$

Moreover, $\quad v([0,1])=\phi(1)+\int_{0}^{1} \alpha d \mu(\alpha)=\int_{0}^{1} \phi(p) d p \quad$ by Fubini's theorem. Thus, under the normalizing constraint $\int_{0}^{1} \phi(p) d p=1, v$ is a probability measure on $[0,1]$ and $M_{\phi}(X)$ appears as a weighted average of expected shortfalls. $\square$

\section{Appendix D: Spectral Risk Measures and Mean-Variance Criterion.}

Proposition D.1. When portfolio risk $X$ is Gaussian, we can write $M_{\phi}(X)$ as: 


$$
M_{\phi}(X)=-E^{P}[X]+\sigma(X) M_{\phi}\left(X_{0}\right)
$$

and $M_{\phi}\left(X_{0}\right) \geq 0$.

Proof of Proposition D.1. Since $X=E^{P}[X]+\sigma(X) X_{0}$ where $X_{0} \sim N(0,1)$, we get:

$$
M_{\phi}(X)=-E^{P}[X]+\sigma(X) M_{\phi}\left(X_{0}\right),
$$

thanks to the positive homogeneity and translation invariance properties of $M_{\phi}$.

In Subsection 2.6, we showed a one-to-one matching between spectral and distortion risk measures. We also established that for any distortion risk measure $\rho_{g}$ we have $\rho_{g}(X) \geq-E^{P}[X]$. Thus the non-negativity of the coefficient $M_{\phi}\left(X_{0}\right)$ can be derived from: $M_{\phi}\left(X_{0}\right) \geq-E^{P}\left[X_{0}\right]=0$.

\section{References}

Acerbi, C., 2002. Spectral measures of risk: a coherent representation of subjective risk aversion. Journal of Banking and Finance 26 (7), 1505-1518

Acerbi, C., Simonetti, P., 2002. Portfolio optimization with spectral measures of risk. Working paper, Abaxbank (Italy).

Acerbi, C., Tasche, D., 2002. On the coherence of expected shortfall. Journal of Banking and Finance 26 (7), 1487-1503.

Agarwal, V., Naik, N.Y., 2000. On taking the 'alternative' route: risks, rewards, style and performance persistence of hedge funds. The Journal of Alternative Investments 2(4), 6-23

Amin, G., Kat, H. M., 2003. Welcome to the dark side: Hedge fund attrition and survivorship bias over the period 1994-2001. Journal of Alternative Investments 6(2), 57-73

Artzner, P., Delbaen, F., Eber, J.-M., Heath, D., 1999. Coherent risk measures. Mathematical Finance.9 (3), 203-228

Bares, P.A., Gibson, R., Gyger, S., 2001. Style consistency and survival probability in the hedge funds' industry. EFA 2001 Barcelone Meetings, EFMA 2001 Lugano Meetings. Available at SSRN or DOI.

Bassett, G. W., Koenker, R., Kordas, G., 2004. Pessimistic portfolio allocation and Choquet expected utility. Journal of Financial Econometrics 2(4), 477-492.

Brooks, C., Kat, H.M., 2002. The statistical properties of hedge fund index returns and their implications for investors. Journal of Alternative Investments 5, 26-44.

Brown, S.J., Goetzmann, W.N., Park, J., 1997. Conditions for survival: changing risk and the performance of hedge fund managers and CTAs. Review of Financial Studies 5, 553-580.

Chabaane, A., Laurent, J.-P., Malevergne, Y., Turpin, F., 2006. Alternative risk measures for alternative investments. Journal of Risk 8(4), 1-32. 
Cheklov, A., Uryasev, S., Zabarankin, M., 2005. Drawdown measure in portfolio optimization. International Journal of Theoretical and Applied Finance 8(1), 13-58.

Cvitanic, J., Lazrak, A., Martellini, L., Zapatero, F., 2003. Optimal allocation to hedge funds: an empirical analysis. Quantitative Finance 3(1), 28-39.

Danielsson, J., Jorgensen, B. N., Sarma, M., De Vries, C., G., 2006. Comparing downside risk measures for heavy tailed distributions. Economic Letters 92(2), 202-208.

Davies, R. J., Kat, H. M., Lu, S., 2003. Fund of hedge funds portfolio selection: a multipleobjective approach. Working paper

De Giorgi, E., 2005. Reward risk portfolio selection and stochastic dominance. Journal of Banking and Finance 29(4), 895-926.

Delbaen, F., 2000. Coherent risk measures. Lecture Notes, Scuola Normale Superiore, Pisa.

Delbaen, F., 2002. Coherent risk measures on general probability spaces. In: Essays in Honour of Dieter Sondermann, Springer-Verlag.

Denneberg, D., 1994. Non-additive measure and integral. Kluwer Academic Publishers, Dordrecht, Germany.

Denneberg, D., 1990. Premium calculation: why standard deviation should be replaced by absolute deviation. ASTIN Bulletin 20(2), 181-190.

Denuit, M., Dhaene, J., Goovaerts, M. J., Kaas, R., Vyncke, D., 2002. The concept of comonotonicity in actuarial science and finance: theory. Insurance: Mathematics and Economics 31(1), 3-33.

Denuit, M., Dhaene, J., Goovaerts, M. J., 2006. Actuarial theory for dependent risks. Wiley.

Dhaene, J., Goovaerts, M. J., Kaas, R., Tang, Q., Vanduffel, S., Vyncke, D., 2004. Capital requirements, risk measures and comonotonicity. Belgian Actuarial Bulletin 4, 53-61.

Embrechts, P., Frey, R., McNeil, A, 2005. Quantitative risk management: concepts, techniques and tools. Princeton University Press.

Fischer, T., 2003. Risk capital allocation by coherent risk measures based on one sidedmoments. Insurance: Mathematics and Economics 32, 135-146.

Fung, W., Hsieh, D.A., 1997. The information context of performance track records: investment style and survivorship bias in historical returns of commodity tracking advisors. The Journal of Portfolio Management 24, 30-41.

Giamouridis, D., Vrontos, I.D., 2007. Hedge fund portfolio construction : A comparison of static and dynamic approaches. Journal of Banking and Finance 31, 199-217. 
Gilli, M., Këllezi, E., 2000. Heuristic approaches for portfolio optimization. In: Sixth international conference on computing in economics and finance of the society for computational economics, Barcelona (Spain).

Gouriéroux, C., Laurent, J.-P., Scaillet, O., 2000. Sensitivity analysis of values at risk. Journal of Empirical Finance 7 (3-4), 225-245.

Gouriéroux, C., Liu, W., 2006. Efficient portfolio analysis using distortion risk measures. Working paper, University of Toronto.

Granger, C., Silvapulle, P., 2001. Large returns, conditional correlation and portfolio diversification: a Value at Risk approach. Quantitative Finance 1 (5), 542-551.

Hahn, C., Pfingsten, A., Wagner, P., 2002. An empirical investigation of the rank correlation between different risk measures. Working paper, Westfalische Wilhelms-Universitat Munster.

Heyde, C.C., Kou, S.G., Peng, X.H., 2006. What is a good risk measure: Bridging the gaps between data, coherent risk measures and insurance risk measures. Working paper, Columbia University.

Jones, B. L., Zitikis, R., 2003. Empirical estimation of risk measures and related quantities. North American Actuarial Journal 7, 44-54.

Jouini, E., Schachermayer, W., Touzi, N., 2006. Law invariant risk measures have the Fatou property, In: Kusuoka, S., Yamazaki, A. (Eds.), Advances in Mathematical Economics, Vol.9, Springer Japan, 49-71.

Kahneman, D., Tversky, A., 1979. Prospect theory: an analysis of decision under risk. Econometrica 47(2), 263-291.

Krokhmal, P., Uryasev, S., Zrazhevsky, G., 2002. Risk management for hedge fund portfolios. Journal of Alternative Investments 5(1), 10-29.

Konno, H., Waki, H., and Yuuki, A., 2002. Portfolio optimization under lower partial risk measures. Asia-Pacific Financial Markets 9(2), 127-140.

Kusuoka, S., 2001. On law invariance coherent risk measures, In: Kusuoka, S., Maruyama, T. (Eds.), Advances in Mathematical Economics, Vol. 3, Springer Japan, 83-95.

Leitner, J., 2005, A short note on second-order stochastic dominance preserving coherent risk measures. Mathematical Finance 15(4), 649-651.

Markowitz, H. M., 1952. Portfolio selection. Journal of Finance 7(1), 77-91.

Morton, D.P., Popova, E., Popova, I., 2006. Efficient fund of hedge funds construction under downside risk measures. Journal of Banking and Finance 30, 503-518.

Panjer, H. H., Wang, S. S., Young, V. R., 1997. Axiomatic characterization of insurance prices. Insurance: Mathematics and Economics 21(2), 173-183. 
Rockafellar, R.T., Uryasev, S., 2000. Optimization of conditional value-at-risk. Journal of Risk 2(3), 21-41.

Rockafellar, R. T., Uryasev, S., 2002. Conditional Value at Risk for general loss distributions. Journal of Banking and Finance 26(7), 1443-1471.

Rockafellar, R. T., Uryasev, S., Zabarankin, M., 2006. Master funds in portfolio analysis with general deviation measures. Journal of Banking and Finance 30, 743-778.

Sharma, M., 2004. AIRAP - Alternative RAPMs for alternative investments. Journal of Investment Management 2(4)

Tasche, D., 2002. Expected shortfall and beyond. Journal of Banking and Finance 26(7), 1519-1533.

Wang, S. S., 2000. A class of distortion operators for pricing financial and insurance risks. Journal of Risk and Insurance 67(1), 15-36.

Yaari, M.-E., 1987. The dual theory of choice under risk. Econometrica 55(1), 95-115 